\title{
Purification Capability of Potted Plants for Removing Atmospheric Formaldehyde
}

\author{
Takashi OYABU, ${ }^{\text {a }}$ Takeshi ONODERA, ${ }^{\mathrm{b}}$ Ayako SAWADA, ${ }^{\mathrm{c}}$ and Kozaburo TAKENAKA ${ }^{\mathrm{d}}$ \\ ${ }^{\mathrm{a}}$ Graduate School of Regional Economic Systems, Kanazawa Seiryo University (10 Gosho-machi, Kanazawa 920-8620, \\ Japan) \\ ${ }^{\mathrm{b}}$ Graduate School of Information Science and Electrical Engineering, Kyushu University (6-10-1 Hakozaki, Higashi-ku, \\ Fukuoka 812-8581, Japan) \\ 'School of Knowledge Science, Japan Advanced Institute Science and Technology (1-1 Asahidai, Tatsunokuchi 923- \\ 1211, Japan) \\ dTakenaka Garden Afforestation Co. Ltd. (7-11-17 Funabori, Edogawa 134-0091, Japan)
}

Received November 15, 2002 ; Accepted March 3, 2003

\begin{abstract}
The purification capabilities of four kinds of foliage plants for formaldehyde, which is a main indoor air pollutant, are examined. The purification capability $\left(P_{a}\right)$ is introduced using a tin oxide gas sensor-output. $P_{a}$ is derived by dividing the peak value of the sensor-output by the full-width at half maximum. Formaldehyde is one of the typical chemical substances which cause sick-building syndrome. The abilities of the following plants are examined: rubber plant, Boston fern, golden pothos and snake plant. The purification capabilities of these plants for formaldehyde in the amount of $10 \mathrm{ppm}$ are about $19,15,23$ and 12 respectively. The number becomes greater as the capability is higher. There are several features in $P_{a}$ characteristics as a function of formaldehyde concentration. Namely, the $P_{a}$ characteristics for rubber plant and Boston fern can be shown by a linear increasing function. The characteristics are measured until the concentration of $40 \mathrm{ppm}$. The characteristic of golden pothos is almost constant and the characteristic of snake plant has a maximum value at the concentration of $20 \mathrm{ppm}$.
\end{abstract}

Key Words : Gas Sensor, Sensory System, Formaldehyde, Sick-building Syndrome

\section{Introduction}

Various types of chemical substances have been accumulating on the earth due to the achievement of high economic growth and the over increasing population. The atmosphere, soil, oceans, lakes and marshes have been polluted by these substances. The polluting chemicals are now one of the major problems on the earth and it takes a vast sum of money to remove the chemicals. Plants, soil and oceans have originally a considerable capability to remove various types of chemicals. It is necessary to investigate these capabilities to construct a future economic policy. Especially, plants are a living thing close to humans and have a high capability to purify the atmospheric chemicals. We should examine the total purifying capability for the exhausted chemicals on the earth and it is necessary to reflect the capability value to our economic activities.

In this study, the purifying capabilities of potted plants for atmospheric chemicals were examined. ${ }^{1)}$ Air pollution is one of the serious problems, especially, indoor air pollution is harmful to human beings because we spend a long term in a domicile. Formaldehyde, toluene and xylene are the harmful indoor air pollutants and are regulated by WHO (World Health Organization). The values of the chemicals in an indoor environment are 0.08, 0.07 and $0.20 \mathrm{ppm}$ respectively. Sick-building syndrome mainly caused by these chemicals. The purification capability of a potted plant for formaldehyde was examined in this study. It is important that people understand the capabilities of the plants to investigate powerfully an environmental problem. A tin oxide gas sensor is adopted to examine the pollution level in the atmosphere. The sensor is widely sold on the market and is used in a gas leakage detector. The sensor resistance decreases in a reducing gas. It becomes lower as the gas concentration becomes higher.

The following plants were employed as the subjects: golden pothos, Boston fern, rubber plant and snake plant. These are typical foliage plants and usually thrive in an indoor environment. The plants are cheap and the maintenance is simple. As for the results, pothos had the highest purification capability out of the examined plants and next is the rubber plant. It became obvious that indoor air pollution could be reduced by a foliage plant.

\section{Experimental}

A plant has a fairly high capability to purify atmospheric pollution if the plant could not damage. It is necessary to understand the type and the concentration of the polluting chemical by using a monitoring technique. Various types of chemicals exist in our surroundings. The cost of the technique must be also considered to detect the pollution. In this study, two types of tin oxide gas sensors are used to indicate the purification capability $\left(P_{a}\right)$ of a plant. ${ }^{2)}$ They have somewhat different characteristics to the indoor air pollutants. One of the charac- 
(a)

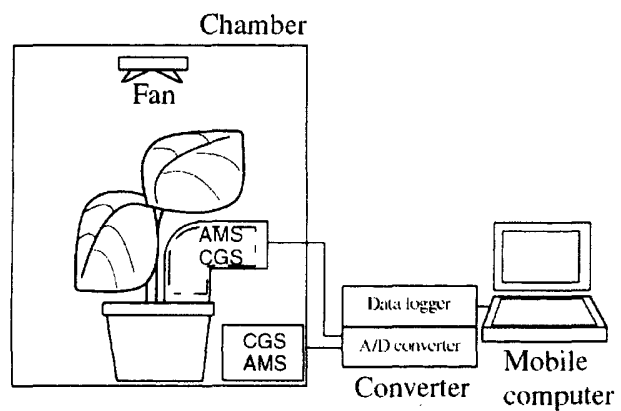

(b)

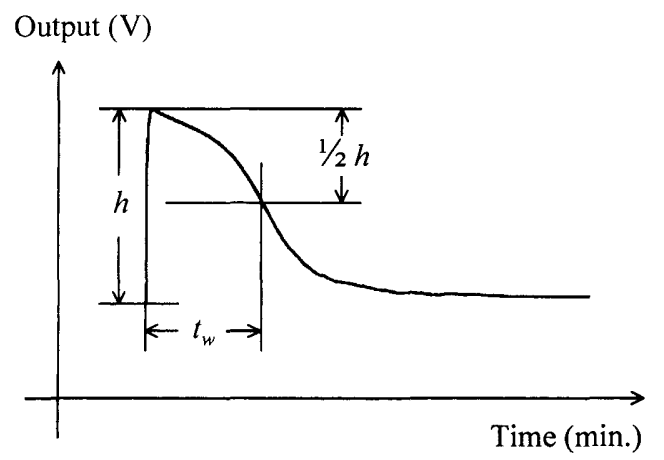

Fig. 1 (a) Measurement system of plant purificationcapability for an indoor air-pollutant. (b) Meaning of the parameters, $h$ and $t_{w}$.

teristics is used to understand the plant purification capabilities according to the type of polluting chemicals. The output of the sensors, which is indicated in voltage, becomes higher as the concentration of polluting chemicals becomes higher. These types of sensors are on the market and are widely used in gas detecting equipment. The sensors also have a high reliability. Recently, it has been shown that the sensor can detect an odor of ppb level. ${ }^{3 \text { ) }}$ The monitoring system using chemical sensors is fairly expected in an indoor environment. The measurement system is shown in Fig. 1(a). ${ }^{2}$

A plant is put up in the experimental chamber as a subject and a polluting chemical is injected into the chamber using a micro syringe. The capacity of the chamber is about 300 liters. Two pairs of two types of sensors are installed in the chamber. One pair is put in the atmosphere of the chamber and another pair is put in the potted soil to monitor the polluting grade in the soil. The polluting chemicals also diffuse into the soil and are purified by various kinds of microorganisms in the soil. Therefore, it is necessary to understand the purification characteristics in the soil. These characteristics were already reported. ${ }^{1)}$ These sensors are named shortly AMS (Ammonia Sensor) and CGS (Combustible Gas Sensor). AMS (Figaro, TGS \#826) has a high sensitivity to ammonia gas and CGS (Figaro, TGS \#800) has a high sensitivity to combustible gases. However, the sensors do not have a high gas-selectivity. Sunlight from outside was isolated in the experimental room and light-intensity was controlled by a fluorescent light, because the sunlight intensity is influenced by the weather and it can not be kept at a constant. Purification capability $\left(P_{a}\right)$ of a plant is influenced by the illumination intensity. $P_{a}$ as a function of the intensity was examined. The temperature in the experimental room was controlled by an airconditioner. The temperature is usually kept at about 22 degrees centigrade by an air conditioner.

Golden pothos, rubber plant, Boston fern and snake plant were employed as subjects. These plants were growing in a pot of $30 \mathrm{~cm}$ diameter and $23 \mathrm{~cm}$ height. The soil of each pot was of the same kind and same quantity including pumice stones of 6.5 liters. $P_{a}$ was derived using the peak value $h$ of AMS sensor characteristics from the base level and the full-width at half maximum $t_{w}$. Namely, $P_{a}$ is indicated by the quation(1). The meaning of the parameters, namely $h$ and $t_{w}$, are shown in Fig. 1(b). The $h$ becomes higher as the concentration of polluting chemicals is higher. The $t_{w}$ becomes shorter as the plant capability is higher. $P_{a}$ becomes high when $t_{w}$ is shorter even though $h$ is high.

$$
P_{a}=h / t_{w} \times 100
$$

The value of $P_{a}$ is proposed to estimate the plant purification-capability for air pollution. It is useful that the capability is indicated using a numerical value.

\section{Results and Discussion}

\section{1 Sensor characteristics}

In the study, the purifying capability is evaluated by AMS sensor characteristic. CGS characteristic will be used to understand the plant capabilities for other chemicals. Each sensor was connected in a series to a load resistance $R_{L}$ and dc power supplier. The voltage of both ends of the load resistance was monitored as an output characteristic. The outputs of four sensors could be input into an A-D converter and data logger $(K E Y E N C E$, NR250 ). After that, the data were processed by a mobile computer as shown in Fig. 1(a). In the first place, it monitored how the AMS output changed when formaldehyde was injected into the chamber. The result is shown in Fig. 2. In the figure, $v$ means the characteristic when a potted plant was not installed in the chamber, namely the chamber was empty. The output $v$ as a function of

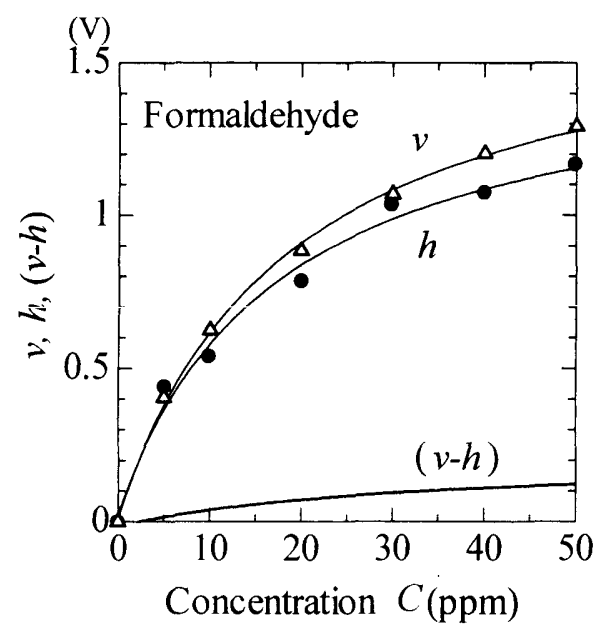

Fig. 2 Output characteristics of a tin oxide gas sensor used in this study as a function of formaldehyde concentration. $v$ means the sensor output, $h$ is a peak of sensor output and $(v$ $h$ ) means the discrepancy in these values. 
formaldehyde concentration $C$ had a tendency that it saturated over $30 \mathrm{ppm}$ of the concentration. The $v$, however, means the output value from the base level of the sensor. The relationships between $v$ and $C$ can be indicated using the following approximate-equation:

$$
v=-32.437 /(C+18.676)+1.749
$$

The characteristic $h$ was monitored when the potted golden pothos was installed in the chamber. The characteristic was also measured as the peak value of the sensor output from the base level. Both characteristics of $v$ and $h$ had a similar tendency and $h$ characteristic was a little lower than $v$ because formaldehyde was adsorbed into the potted plant and diffused into the soil. The approximate equation for the $h$-characteristic is expressed using the equation (3). The value of $(v-h)$ means a diffused or absorbed amount at each concentration. The characteristic has a tendency that the value saturates at high concentration of formaldehyde. The approximate function of the characteristic is indicated in the equation (4). This equation is peculiar to this potted pothos.

$$
\begin{gathered}
h=-26.626 /(C+17.463)+1.549 \\
(v-h)=-7.499 /(C+33.031)+0.213
\end{gathered}
$$

It became obvious from Fig. 2 and eq. 4 that the formaldehyde concentration of about only $1 \mathrm{ppm}$ was absorbed into the potted plant and/or diffused into the soil at injection time.

\section{2 Purification characteristics}

Purification characteristics for four kinds of potted plants are shown in the following figures. These characteristics were examined at the atmospheric formaldehyde of $10 \mathrm{ppm}$. Fig. 3 is for the rubber plant, Fig. 4 is for Boston fern, Fig. 5 is for golden pothos and Fig. 6 is for snake plant. In these figures, the sensor characteristics in soil were not shown. The characteristics were already reported ${ }^{11}$ and the characteristics of this study had almost same tendencies with them. The concentration in soil became until the level of $1 \mathrm{ppm}$ when the volume of $10 \mathrm{ppm}$ was injected into the chamber of 300 litters. In these experiments, the sprinkling of water was done at 8 : $30 \mathrm{am}$ and the door of the chamber was closed. The injection of formaldehyde was done at $12: 30 \mathrm{pm}$. These processes were done in every experiment. The characteristics in every figure are shown from 1 hour before the injection. The characteristics immediately increased about $0.6 \mathrm{~V}$ after the injection of formaldehyde, and airquality in the chamber became purified by the subjective plant. This phenomenon was monitored by the sensor outputs. A part of the experimental pollutant was adsorbed by the potted plant and/or diffused into the soil. Therefore the peak levels in these figures have some differences. Each characteristic reached the peak level and returned to the base level after about 10 hours. But the level was a little high compared with the base level due to high humidity by the transpiration of the plant in the chamber and it was considered that various kinds of very low-concentration gases occurred from the potted plant.

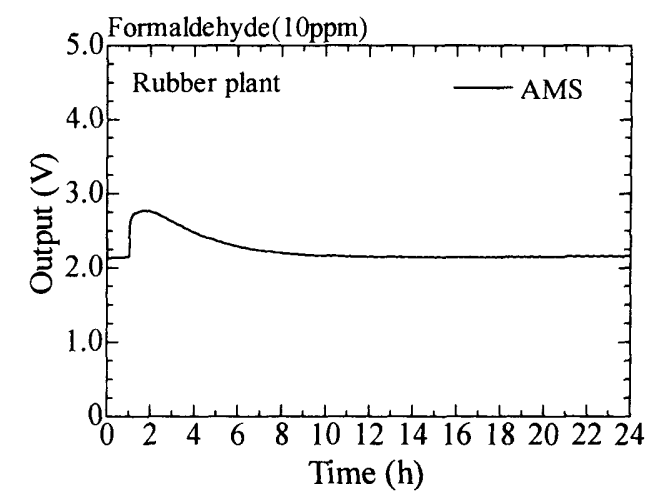

Fig. 3 Purification capability of a rubber plant for $10 \mathrm{ppm}$ of formaldehyde under a light.

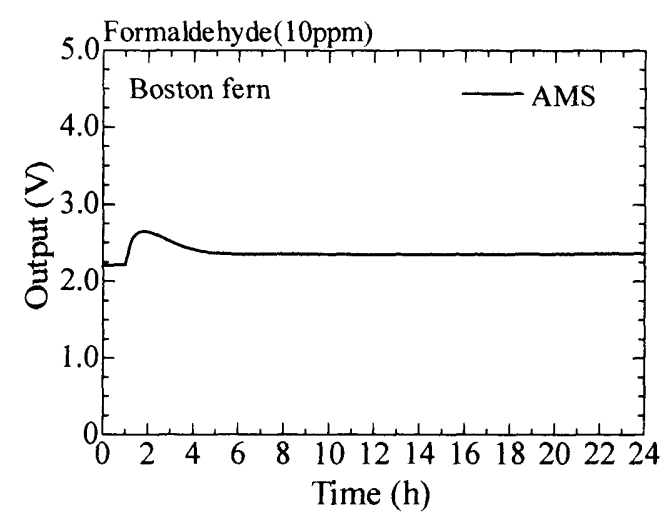

Fig. 4 Purification capability of a Boston fern for $10 \mathrm{ppm}$ of formaldehyde under a light.

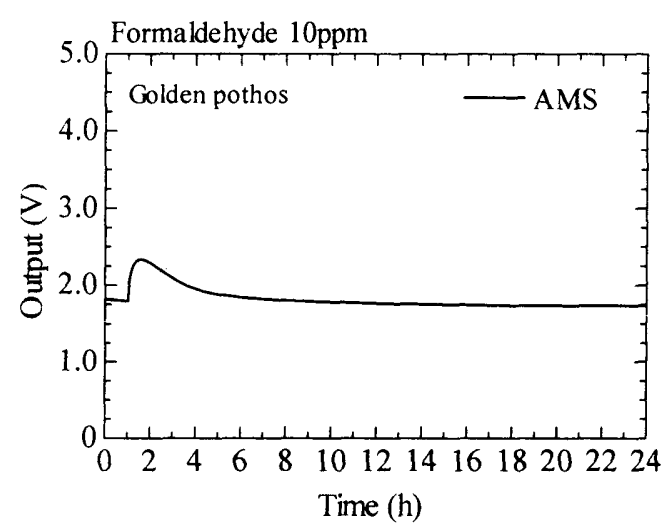

Fig. 5 Purification capability of a golden pothos for $10 \mathrm{ppm}$ of formaldehyde under a light.

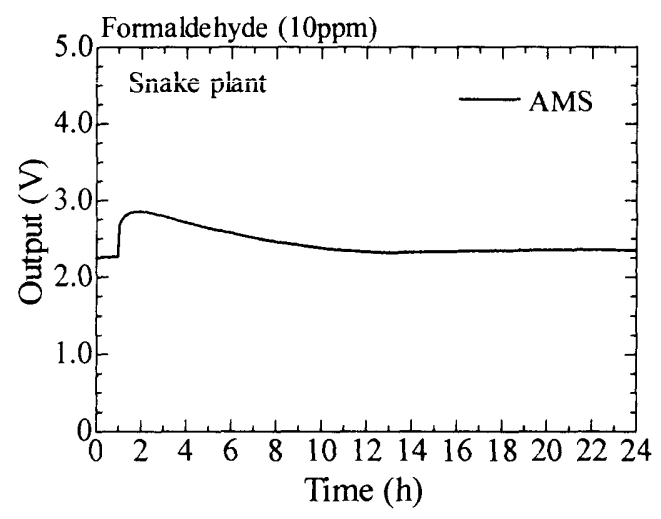

Fig. 6 Purification capability of a snake plant for $10 \mathrm{ppm}$ of formaldehyde under a light. 
The gases are not harmful. The concentration of formaldehyde was under $0.04 \mathrm{ppm}$ at the base level. The value was evaluated by a gas chromatography.

The sensor characteristic reached a peak level in a few minutes when the atmosphere was stirred up by the fan installed at the top of the chamber. It reached this level in 20 minutes after the injection of formaldehyde when the fan was not used. Therefore, it is thought that it takes about 20 minutes to make uniform concentration unartificially. The sensor characteristic reached the peak level and decreased slowly because formaldehyde was taken into the plant organ through the stomata and the slight volume diffused into the soil of the pot. At that time, the concentrations of carbon dioxide was varied in the range of 280 to $450 \mathrm{ppm}$ and oxygen were almost constant (about $20.9 \%$ ). It is, however, necessary to examine both concentrations using a precision instrument.

\section{3 Purification capability of plants}

Purification capability $\left(P_{a}\right)$ of a plant is defined using the equation (1). $P_{a}$ s for four kinds of potted plants are summarized in Table 1. Each $P_{a}$ was evaluated under formaldehyde of $10 \mathrm{ppm}$. The suitable thriving temperaturerange of each plant is shown in the table and the ranges are nearly similar. Golden pothos had the highest capability and snake plant was the lowest. There were about twice differences in these $P_{a}$ s. We can not conclude that leafy plants have a high purification capability. $P_{a}$ values indicated in Table 1 changed a little according to plant metabolism, circadian rhythm and the season. But the order does not change. These experiments were carried out several. At the present time, it is difficult to examine and conclude which factors, for example, the variety of plant, the size of plant leave and age, strongly influence the $P_{a}$. The $P_{a}$ of each plant as a function of formaldehyde concentration was examined. The results are shown in Fig. 7 to 10. Fig. 7 shows the $P_{a}$ characteristic for the rubber plant. Fig. 8 shows the characteristic of the Boston fern and Fig. 9 is for golden pothos. Fig. 10 is for snake plant. The $P_{a}$ characteristics for rubber plant and Boston fern became larger gradually as the formaldehyde concentration became higher.

Most plants are damaged in atmospheric chemicals over $50 \mathrm{ppm}$ and those sometimes have spotted leafepidermis. Therefore, the experiments were carried out bellow $50 \mathrm{ppm}$. Boston fern has many leaves and the total area of all leaves is larger than that of rubber plant. But $P_{a}$ of Boston fern is a little lower than that of rubber plant. Consequently, it can be estimated that $P_{a}$ does not depend on leaf area. ${ }^{4} P_{a}$ characteristic of golden pothos

Table 1 Purification-capability $\left(P_{a}\right)$ of the examined plants. The specific forms, the habitats and growing temperature are also indicated.

\begin{tabular}{ccccc}
\hline Plant & Specific forms & Habitat & Temperature $\left({ }^{\circ} \mathrm{C}\right)$ & $\mathrm{Pa}$ \\
\hline Rubber plant & maraceae & India, Malaysia & $16 \sim 27$ & 19 \\
Boston fern & & Mexico, Jamaica & $10 \sim 24$ & 15 \\
Golden pothos & \multirow{2}{*}{ araceae } & Solomon Is. & $18 \sim 24$ & 23 \\
Snake plant & & West Africa & $18 \sim 27$ & 12 \\
\hline
\end{tabular}

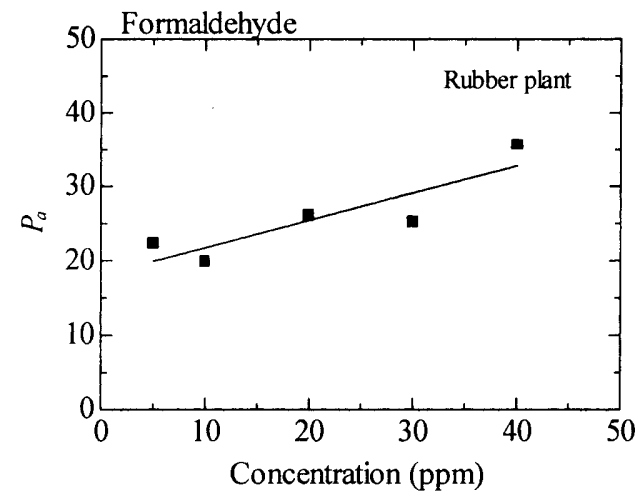

Fig. 7 Purification characteristics of the rubber plant as a function of formaldehyde concentration.

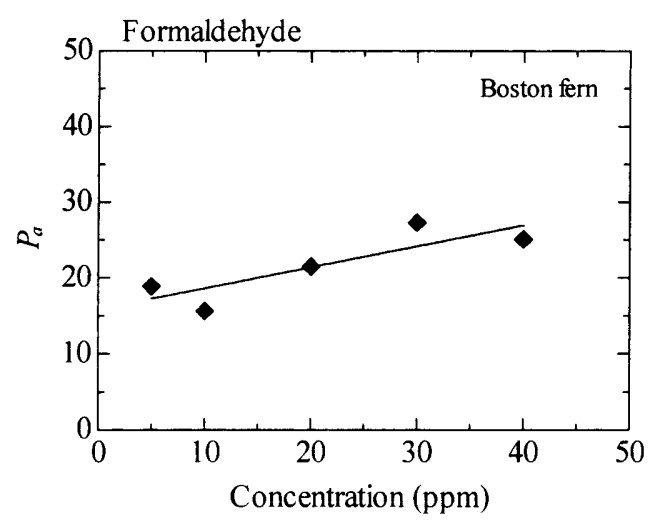

Fig. 8 Purification characteristics of the Boston fern as a function of formaldehyde concentration.

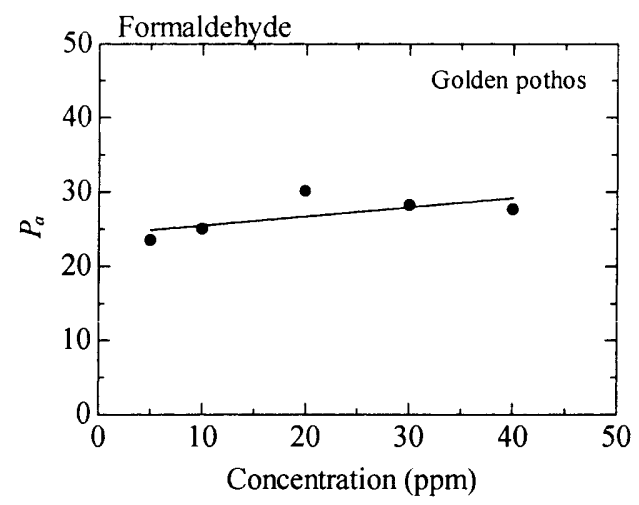

Fig. 9 Purification characteristics of the golden pothos as a function of formaldehyde concentration.

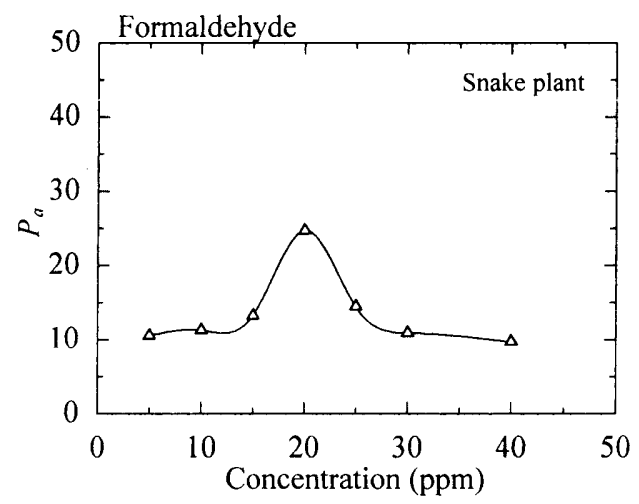

Fig.10 Purification characteristics of the snake plant as a function of formaldehyde concentration. 


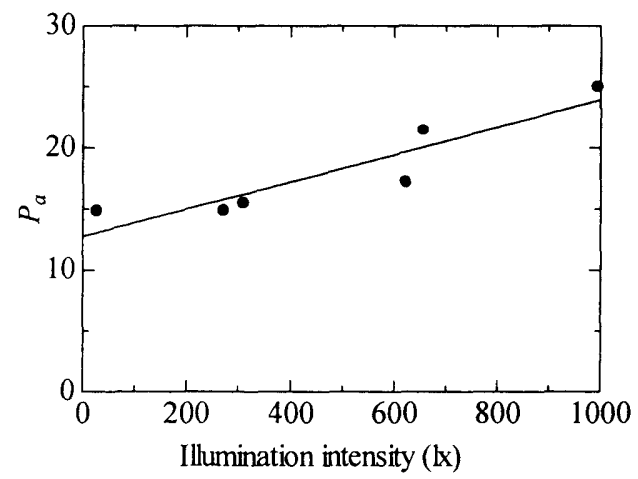

Fig. 11 Purification capability as a function of illumination intensity. The characteristic becomes saturated over the intensity of $1000 l x$.

had nearly a constant value of 25 as shown in Fig. 9 and it will be easier to introduce a characteristic of an indoor air-quality when a golden pothos is put in a room. In Fig. 10 , for a snake plant, the $P_{a}$ characteristic has a peak value at $20 \mathrm{ppm}$. The plant had a peak value at that concentration in several experiments and the range of $P_{a}$ was 22 to 24.5. $P_{a}$ is fairly influenced by soil-bacteria and the biorhythm of the plant. There is an ecosystem among the plant and soil bacteria living in the rhizospheres.

\section{4 Effects of light intensity}

The purification characteristic is usually influenced by light intensity. The capability characteristic as a function of illumination intensity is shown in Fig. 11. The capabilities in the figure were evaluated at a formaldehyde concentration of $30 \mathrm{ppm}$. In Fig. 11, $P_{a}$ becomes higher little by little as the intensity becomes higher. ${ }^{5,6)}$ It is thought that the capability relates to the photosynthesis of the plant and high purification capability is taken at high intensity. ${ }^{7,8)}$ The capability saturated over $1000 \mathrm{~lx}$. The plant had a $P_{a}$ of 15 in darkness. ${ }^{2)}$ The characteristic of Fig. 11 is shown by using the following equation:

$$
P_{a}=0.0111 \times l+12.76
$$

The variable $l$ means light intensity $(l x)$. We can derive the purification capability for light intensity using the equation and also estimate the air quality in a room which contains the plant. It is useful to estimate the air quality in an indoor environment. It is better to put the plant in a proper intensity of sunlight.

It was also obvious that the pothos had a maximum $P_{a^{-}}$ value at an atmospheric temperature of 22 degrees centigrade.

\section{Conclusions}

The purification capabilities $\left(P_{a}\right)$ of four kinds of plants for formaldehyde as a pollutant were examined using tin oxide gas sensors. Rubber plant, Boston fern, golden pothos and snake plant were used as the subjective plants. $P_{a}$ was derived by dividing the peak value of the sensoroutput by the full-width at half maximum. The peak value becomes higher as the polluting level becomes higher and the width becomes narrower as the plant has higher capability. The sensor has a high reliability and is easy to obtain. As for the results, there are differences in the purification capability for the same size of plants and it becomes obvious that each $P_{a}$ characteristic as a function of formaldehyde concentration has own feature. The $P_{a} \mathrm{~s}$ took the values of about 10 to 25 in the examined plants.

It will be necessary to examine the influences of light intensity, the sprinkling cycle and the kind of soil to $P_{a}$. And its difference to the type of pollutant must be also investigated.

\section{Acknowledgments}

The authors thank Dr. Yuki Hasegawa and Prof. Teruaki Katsube of Saitama University for their useful advices, and thank Dr. B.C. Wolverton for the technical discussions.

\section{References}

1) T. Oyabu, T. Onodera, H. Kimura, and Y. Sadaoka, J. Jpn. Soc. Atmos. Environ., 34, 319 (2001).

2) T. Oyabu, A. Sawada, T. Onodera, K. Takenaka, and Bill Wolverton, Sensors and Actuators B, 89, 131 (2003).

3) T. Oyabu, T. Misawa, H. Kimura, and H. Nanto, MATERIAL SCIENCE \& ENGINEERING C: Biomimetic Materials, Sensors and Systems, 12, 89 (2000).

4) W. Wang, J. W. Gorsuch, and J. S. Hughes, Plant for Environmental Studies, LEWIS PUBLISHERS (1997).

5) B.C.Wolverton and J. D.Wolverton, J. Missi. Acad. Sci., 38, 11 (1993).

6) T. Kondo, K. Hasegawa, R. Uchida, M. Onishi, A. Mizukami, and K. Omasa, Bull. Chem. Soc. Jpn., 69, 3673 (1996).

7) B. J. Nebel and R. T. Wright, Environmental Science, Prentice Hall (New Jersey, 2000).

8) L. Brussaard and R. Ferrera-Cerrato, Soil Ecology in Sustainable Agricultural Systems, Lewis Publishers (New York, 1997). 